\title{
Current Trends in Neuropathology
}

Editor

DAVID M. MEREDITH

\section{SURGICAL PATHOLOGY CLINICS}

www.surgpath.theclinics.com

Consulting Editor

JASON L. HORNICK

June 2020 - Volume 13 - Number 2 